\title{
Tratamento de linfocele inguinal pós-operatória com injeção de cola de fibrina: relato de caso
}

\author{
Treatment of postoperative inguinal lymphocele with fibrin glue \\ injection: case report
}

\author{
Priscila Nunes Boaventura, Marcone Lima Sobreira, Winston Bonetti Yoshida, Hamilton Almeida Rollo*
}

\begin{abstract}
Resumo
O paciente desenvolveu linforragia na região inguinal direita, depois de ponte aorto-bifemoral com enxerto de dácron ${ }^{\circledR}$. Não respondeu ao tratamento conservador com cuidados locais e compressão. Foi realizado exame de ultra-sonografia Doppler, que evidenciou grande linfocele $(6,4 \times 3,36 \times 6,1 \mathrm{~cm})$, tratada pelo esvaziamento da loja por aspiração e injeção de cola de fibrina $(1,6 \mathrm{~mL})$ em seu interior. $\mathrm{O}$ paciente se recuperou sem intercorrências e sem recidiva, após 3 meses de seguimento.
\end{abstract}

Palavras-chave: Linfocele, adesivo tecidual de fibrina, cirurgia vascular.

\section{Introdução}

A linforragia inguinal decorrente da lesão de vasos linfáticos é uma complicação freqüente após a exposição da artéria femoral comum em pacientes submetidos à revascularização infrainguinal ${ }^{1}$, que costuma ocorrer em 0,8 a $6,4 \%$ dos $\operatorname{casos}^{2}$. Freqüentemente, a linfa não extravasada fica contida pelos tecidos vizinhos, e o processo inflamatório desencadeado pela sua presença favorece o desenvolvimento de uma cápsula ao seu redor, dando origem a uma linfocele. Em geral, as pequenas linfoceles regridem espontaneamente dentro de 2 a 3 dias, podendo ser acompanhadas clinicamente ${ }^{1}$. Entretanto, o aumento da pressão linfática, inflamação, infecção e a presença de próteses podem levar ao aumento de volume das mesmas, tornando imperioso o seu tratamento para evitar a infecção da ferida operatória e da restauração vascular local.

\begin{abstract}
The patient developed lymphatic drainage in the right groin after an aortobifemoral bypass with Dacron ${ }^{\mathbb{B}}$ graft. Conservative treatment with local wound care and compression was unsuccessful. Duplex scan showed a $6.4 \times 3.36 \times 6.1 \mathrm{~cm}$ lymphocele, which was treated by aspiration and injection of fibrin glue $(1.6 \mathrm{~mL})$. The patient recovered uneventfully and without recurrence after a 3-month follow-up.
\end{abstract}

Keywords: Lymphocele, fibrin tissue adhesive, vascular surgery.

Alguns estudos mostraram sucesso do uso da cola de fibrina no tratamento de pseudo-aneurismas inguinais após punções e cateterismos femorais ${ }^{3,4}$. Outros estudos mostraram também o uso da cola de fibrina na prevenção e tratamento de complicações linfáticas, principalmente após linfadenectomias radicais, como também a aparente vantagem em reduzir a incidência de infecção de sítio cirúrgico em pós-operatório $(\mathrm{PO})^{5-7}$.

O objetivo deste relato de caso é mostrar a eficácia da cola de fibrina como opção de tratamento de linforragia e linfocele, após cirurgia de abordagem da artéria femoral na região inguinal.

\section{Relato do caso}

Paciente de 75 anos de idade, sexo masculino, hipertenso, dislipidêmico, ex-tabagista e ex-etilista. Há 5 anos vinha apresentando claudicação intermitente

* Hospital das Clínicas, Faculdade de Medicina de Botucatu, Universidade Estadual Paulista Júlio de Mesquita Filho, Botucatu, SP.

Artigo submetido em 08.03.07, aceito em 21.05.07. 
para $300 \mathrm{~m}$, que piorou para $50 \mathrm{~m}$ acompanhada de aparecimento espontâneo de lesão trófica em $5^{\circ}$ pododáctilo direito, sem cicatrização há 7 meses. Ao exame físico, ausência de todos os pulsos dos membros inferiores. O Doppler mostrava ausência de sinal de fluxo sanguíneo em artérias poplítea e distais bilateralmente. A arteriografia evidenciou oclusão em território aortoilíaco.

Foi submetido a enxerto aorto-bifemoral com prótese de dácron ${ }^{\circledR}$ bifurcada, de $16 \times 8 \mathrm{~mm}$, apresentando no $11^{\circ} \mathrm{PO}$ drenagem espontânea de secreção espessa e purulenta em região inguinal direita, associada a hiperemia de incisão cirúrgica. Foi realizada cultura da secreção, que evidenciou Staphylococcus aureus sensível à vancomicina ${ }^{\circledR}$. Após início da antibioticoterapia, houve melhora expressiva da infecção, mas não da drenagem local. No $24^{\circ} \mathrm{PO}$, apresentava abaulamento em região inguinal direita, com saída espontânea de linfa através da cicatriz cirúrgica. A ultra-sonografia Doppler evidenciou coleção de densidade líquida e sem fluxo em região inguinal direita $(6,4 \mathrm{~cm} \times 3,36 \mathrm{~cm} \times 6,1$ $\mathrm{cm})$, sendo diagnosticado linfocele. Empregando-se a técnica usada para fechamento de pseudo-aneurismas arteriais, optou-se por tentativa de fechamento do orifício de drenagem guiado por ultra-sonografia: assepsia local + expressão manual + punção aspirativa (cerca de $40 \mathrm{~mL}$ ) com esvaziamento da loja e injeção de $1,6 \mathrm{~mL}$ de cola de fibrina (Beriplast ${ }^{\circledR} P-Z L B$ Behring) na loja, seguida de compressão manual por 15 minutos e colocação de peso local, que permaneceu por 24 horas. Após este período, observou-se não haver abaulamentos ou drenagem linfática local (espontânea ou à expressão local). Repetida a ultra-sonografia Doppler, visualizou-se pequena loja em região distal da incisão cirúrgica, porém sem comunicação com o orifício de drenagem prévio. Realizou-se, então, nova punção local e aspiração de $10 \mathrm{~mL}$ de linfa clara e sem grumos com esvaziamento da loja e orientado curativo compressivo. Não houve formação de nova coleção ou desenvolvimento de sinais flogísticos locais.

Após 90 dias de PO, permaneceu assintomático, sem abaulamento (Figura 1) ou drenagem de linfa em cicatriz cirúrgica direita e sem sinais flogísticos locais. A ultra-sonografia Doppler de controle não evidenciou coleção no local (Figura 2).

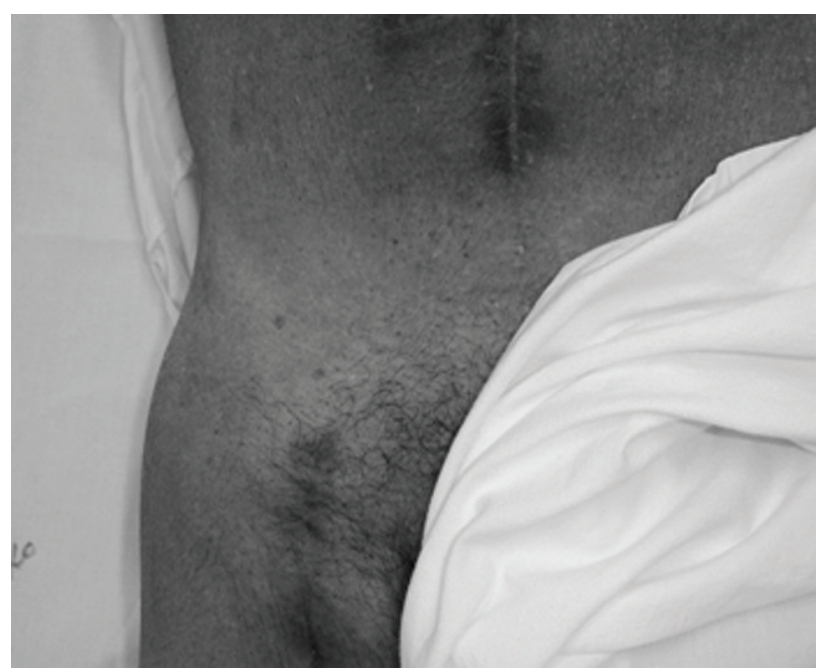

Figura 1 - Ausência de abaulamento em região inguinal direita no $90^{\circ}$ pós-operatório

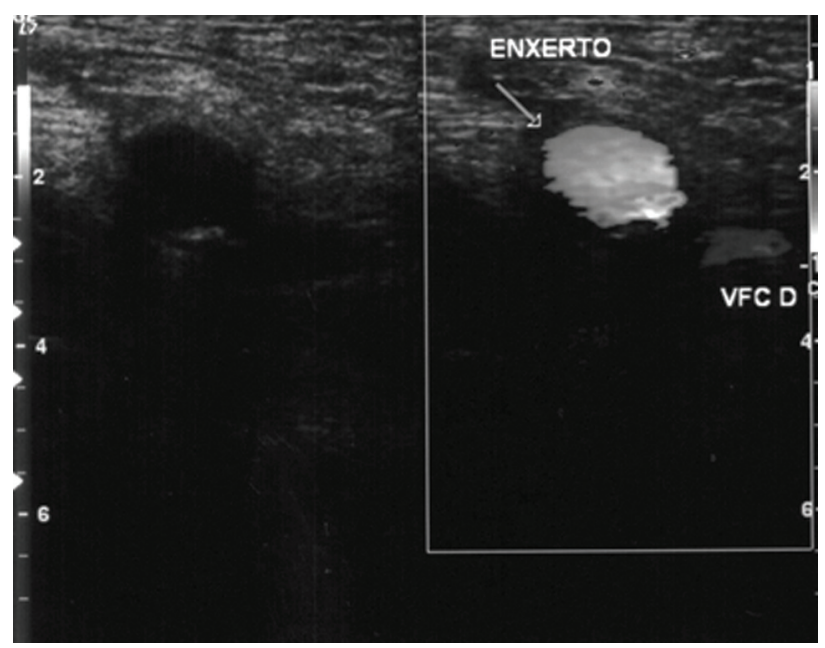

Figura 2 - Enxerto de dácron e veia femoral comum direita sem evidência de massa ou coleção

\section{Discussão}

O tratamento das linfoceles que não reabsorvem clinicamente é, em geral, cirúrgico ${ }^{2}$. As possibilidades de intervenção cirúrgica variam de drenagem cirúrgica e cauterização dos vasos linfáticos a excisão linfática cirúrgica e ligadura de vasos linfáticos ${ }^{8}$. 
A cola de fibrina tem sido usada com sucesso no fechamento de pseudo-aneurismas da artéria femoral após punções ou cateterismos ${ }^{3,4,9}$, ou mesmo em anastomoses arteriais ${ }^{10}$. Alguns estudos mostraram também a utilização da cola de fibrina na prevenção e tratamento de complicações linfáticas, principalmente após linfadenectomias radicais. Giovannacci et al. realizaram estudo prospectivo em pacientes submetidos a revascularizações arteriais, obtendo menor número de complicações linfáticas no grupo em que foi utilizada a cola de fibrina $(\mathrm{p}=0,019)^{1}$.

A linfa tem componentes que promovem a sua coagulação. Neste sentido, a introdução de substâncias pró-coagulantes poderia acelerar este processo, sendo útil para reduzir a drenagem, evitando assim intervenções cirúrgicas maiores para o paciente, o que aumentaria a morbidade. Além deste efeito pró-coagulante, um provável efeito de massa da cola (depois de constituída) exerceria um tampão sobre os orifícios de drenagem da linfocele, contribuindo para a redução e cessação da linforragia.

O sucesso da terapêutica com a cola de fibrina depende também da compressão local, inicialmente manual, e depois mantida com peso inguinal. Este tipo de tratamento para linfocele pós-cirurgia vascular com abordagem femoral não foi encontrado na literatura. Entretanto, em vigência de sinais sugestivos de infecção na área de linfocele, acreditamos que, de forma equivalente ao pseudo-aneurisma arterial, o tratamento com injeção de cola de fibrina está também contra-indicado, devido à possibilidade de manutenção do agente infeccioso no sítio.

Em suma, sugerimos que, em situações onde a presença de linforragia possa prejudicar ou comprometer a revascularização realizada, pode-se optar pelo esvaziamento da loja seguido de injeção de cola de fỉbrina + compressão local, lembrando que, na maioria dos casos, a utilização de técnica operatória cuidadosa e cuidados pós-operatórios podem ser suficientes para evitar o surgimento de complicações como esta.

\section{Referências}

1. Giovannacci L, Renggli JC, Eugster T, Stierli P, Hess P, Gürke L. Reduction of groin lymphatic complications by application of fibrin glue: preliminary results of a randomized study. Ann Vasc Surg. 2001;15:182-5.

2. Pereira AH, Grudtner MA. Complicações não-infecciosas da cirurgia arteriais. In: Brito CJ, Duque A, Merlo I, Murilo R, Fonseca Filho VL. Cirurgia vascular. Rio de Janeiro: Revinter; 2002. p. 768-9.

3. Sadiq S, Ibrahim W. Thromboembolism complicating thrombin injection of femoral artery pseudoaneurysm: management with intraarterial thrombolysis. J Vasc Interv Radiol. 2001;12:633-6.

4. Morgan R, Belli AM. Current treatment methods for postcatheterization pseudoaneurysms. J Vasc Interv Radiol. 2003;14:697-710.

5. Waclawiczek HW, Pimpl W. [Lymph fistulae following lymph node dissections - prevention and treatment using fibrin glue]. Chirurg. 1986;57:330-1.

6. Furrer M, Inderbitzi R, Nachbur B. [Does administration of fibrin glue prevent development of lymphoceles after radical lymphadenectomy?] Chirurg. 1993;64:1044-9.

7. Gilly FN, Francois Y, Sayag-Beaujard AC, Glehen O, Brachet A, Vignal J. Prevention of lymphorrhea by means of fibrin glue after axillary lymphadenectomy in breast cancer: prospective randomized trial. Eur Surg Res. 1998;30:439-43.

8. Shermak MA, Yee K, Wong L, Jones CE, Wong J. Surgical management of groin lymphatic complications after arterial bypass surgery. Plast Reconstr Surg. 2005;115:1954-62.

9. Pinto DM, Dias Jr. JO, Fonseca BLC, Moreialvar RD, Bez LG, Lopes CS. Experiência inicial com o uso de adesivo tissular contendo trombina para tratamento do pseudo-aneurisma femoral. J Vasc Bras. 2006;5:30-6.

10. Yoshida WB, Naresse LE, Rodrigues AC, Fabris VE, Angeleli AY. End-to-end arterial anastomosis with fibrin glue in larger arteries: histology, hydroxyproline concentration and tensile strength study in carotids of rabbits. Acta Cir Bras. 2002;17: 4-11.

\section{Correspondência}

Priscila N. Boaventura

Departamento de Cirurgia e Ortopedia da Faculdade de Medicina de Botucatu

Distrito de Rubião Júnior, $\mathrm{s} / \mathrm{n}^{\circ}$

CEP 18618-970 - Botucatu, SP

Tel.: (14) 3811.6305

E-mail: priboaventura@gmail.com 